\title{
Pharmacokinetic-Pharmacodynamic Relationship of Erenumab (AMG 334) and Capsaicin-Induced Dermal Blood Flow in Healthy and Migraine Subjects
}

\author{
Thuy $\mathrm{Vu}^{\prime}$ • Peiming $\mathrm{Ma}^{2}$ • Jiyun Sunny Chen ${ }^{3}$ • Jan de Hoon ${ }^{4}$ • Anne Van Hecken ${ }^{4}$. \\ Lucy Yan' $\cdot$ Liviawati Sutjandra Wu' $\cdot$ Lisa Hamilton ${ }^{5} \cdot$ Gabriel Vargas'
}

Received: 6 January 2017 / Accepted: 15 May 2017 / Published online: 7 June 2017

(C) The Author(s) 2017. This article is an open access publication

\begin{abstract}
Purpose Capsaicin-induced dermal blood flow (CIDBF) is a validated biomarker used to evaluate the target engagement of potential calcitonin gene-related peptide-blocking therapeutics for migraine. To characterize the pharmacokinetics (PK) and quantify the inhibitory effects of erenumab (AMG 334) on CIDBF, CIDBF data were pooled from a single- and a multiple-dose study in healthy and migraine subjects.
\end{abstract}

Methods Repeated capsaicin challenges and DBF measurements were performed and serum erenumab concentrations determined. A population analysis was conducted using a nonlinear mixed-effects modeling approach. Effects of body weight, gender, and age on model parameters were evaluated. Results Two-compartment target-mediated drug disposition (TMDD) model assuming binding of erenumab in the central compartment best described the nonlinear PK of erenumab. Subcutaneous absorption half-life was 1.6 days and bioavailability was $74 \%$. Erenumab produced a maximum inhibition of $89 \%$ (95\% confidence interval: 87-91\%). Erenumab concentrations required for $50 \%$ and $99 \%$ of maximum inhibition were $255 \mathrm{ng} / \mathrm{mL}$ and $1134 \mathrm{ng} / \mathrm{mL}$, respectively.

Electronic supplementary material The online version of this article (doi: | 0. I007/s | | 095-0 | 7-2 | 83-6) contains supplementary material, which is available to authorized users.

Thuy Vu

thuy@amgen.com

Clinical Pharmacology, Modeling and Simulation, Amgen Inc., One Amgen Center Drive, Thousand Oaks, California 91320-1799, USA

2 Clinical Pharmacology, GSK R\&D, Shanghai, China

3 Medivation, San Francisco, California, USA

4 Center for Clinical Pharmacology, University Hospitals of Leuven, Leuven, Belgium

5 Global Biostatistical Sciences, Amgen Limited, Uxbridge, England, UK
Increased body weight was associated with increased erenumab clearance but had no effect on the inhibitory effect on CIDBF.

Conclusions Our results show that erenumab pharmacokinetics was best characterized by a TMDD model and resulted in potent inhibition of CIDBF.

KEY WORDS anti-CGRP receptor · dermal blood flow · migraine $\cdot$ pharmacokinetics-pharmacodynamics $\cdot$ vasodilation

\section{ABBREVIATIONS \\ CGRP Calcitonin gene-related peptide \\ CIDBF Capsaicin-induced dermal blood flow \\ DBF Dermal blood flow \\ $\mathrm{IC}_{50} \quad$ Concentration providing $50 \%$ inhibitory effect \\ IC99 Concentration providing 99\% inhibitory effect \\ IV Intravenous \\ MAD Multiple-ascending dose \\ PD Pharmacodynamic \\ PK Pharmacokinetic \\ SAD Single-ascending dose \\ SC Subcutaneous \\ TMDD Target-mediated drug disposition \\ TRPVI Transient receptor potential vanilloid type 1 receptor \\ VPC Visual predictive check}

\section{INTRODUCTION}

Migraines are episodic headaches that are sometimes preceded by sensory warning symptoms or signs (auras) and are often accompanied by nausea, vomiting, and extreme sensitivity to light (photophobia) and sound (phonophobia). There is a great unmet medical need for effective migraine therapies, especially for prophylactic treatment. Over 36 million Americans 
$(12 \%$ of the population of the United States) suffer from migraine attacks, with approximately 4 million patients enduring severe impairment and requiring bed rest for 4 or more days per month. Results from recent large-scale headache questionnaires indicate that approximately 14 million migraineurs in the United States would qualify for and benefit from an effective and safe preventive therapy [1-3]. Globally, it is believed that migraine affects more than $10 \%$ of the world's population and causes substantially more individual morbidity [4]. The debilitating effects of migraine have a substantial economic impact on the individual and society, and cause significant social and school-/work-related losses.

Calcitonin gene-related peptide (CGRP) is a neuropeptide and vasodilator, which has been implicated as a key mediator in the initiation and progression of migraine pain. Results from clinical studies with small molecule CGRP-antagonists (e.g., olcegepant and telcagepant) as well as monoclonal antibodies (mAbs) neutralizing CGRP or blocking its receptor, have demonstrated that targeting the CGRP pathway provides clinical benefit to individuals with migraine. Given the efficacy of CGRPblocking therapeutics both in the treatment of acute migraine pain and in prevention, the so-called capsaicin model was developed to assess target engagement for the evaluation of these compounds in early clinical development. The capsaicin model involves the topical application of capsaicin on the human forearm skin. By activating the transient receptor potential vanilloid type 1 (TRPV1) receptor on nociceptive nerve endings, capsaicin induces the local release of CGRP and increases the dermal blood flow (DBF), which can be measured using laser Doppler imaging. The capsaicin-induced DBF (CIDBF) model is well validated, non-invasive, and technically uncomplicated [5]. As it has previously been shown to be a robust model and allows repeated measurements which are reproducible over time, it facilitates the early clinical evaluation of CGRP-blocking therapeutics [6].

Erenumab (AMG 334) [7] is a mAb that binds to the CGRP receptor and thus functions as a competitive inhibitor of the native ligand CGRP. In vitro data have shown that erenumab binds to human CGRP receptors with high affinity (dissociation equilibrium constant $\mathrm{K}_{\mathrm{D}}=20 \mathrm{pM}$ ) and potency $\left(\mathrm{IC}_{50}=2.3 \mathrm{nM}\right)$ in a competitive and reversible manner [8]. Results from single-ascending dose (SAD) and multipleascending dose (MAD) phase 1 studies (NCT01688739 and NCT01723514, respectively) have been reported and showed that erenumab inhibited CIDBF in healthy and migraine subjects, indicating CGRP-receptor antagonism [9] (de Hoon JN, Van Hecken A, Yan L, Smith B, Chen JS, Bautista E, et al., unpublished data). Results from non-compartmental analysis suggested nonlinear pharmacokinetic (PK) characteristics for erenumab; namely, a decreased clearance with increased dose, which is typical of target-mediated drug disposition (TMDD) for mAb therapeutics [10, 11$]$.

This article reports the results of a population PKpharmacodynamics (PD) analysis that quantified the relationship between serum erenumab concentrations and inhibition of CIDBF in healthy subjects and migraine patients. The objectives of this analysis were to: 1) characterize the time course of serum erenumab concentrations following subcutaneous (SG) and intravenous (IV) administrations using a TMDD model; 2) quantify the inhibitory effect of systemic exposure of erenumab on CIDBF; and 3) evaluate the effect of baseline characteristics and other variables as potential sources of variability in $\mathrm{PK}$ and $\mathrm{PD}$ parameters for erenumab. The modeling results would be used to design rational dose regimens for a phase 2 study evaluating safety and efficacy of erenumab in the target population of patients with migraine.

\section{MATERIALS AND METHODS}

\section{Study Design}

PK and DBF data were pooled from a SAD study and a MAD study for population PK-PD analysis. Inclusion/exclusion criteria and assessments for these two clinical studies have been reported $[12,13]$. The SAD study was a double-blind, placebo-controlled, sequential dose escalation study, in which 60 subjects were enrolled. Forty-eight healthy subjects were randomized in a $3: 1$ ratio (erenumab:placebo) to receive erenumab or placebo, where the erenumab treatment was either SC administration of 1, 7, 21, 70, 140, or $210 \mathrm{mg}$ or IV of $140 \mathrm{mg}$. In addition, a total of 12 migraine subjects were randomized in a 1:1 ratio to receive a $\mathrm{SC}$ dose of $140 \mathrm{mg}$ erenumab or placebo. The MAD study was a double-blind, placebo-controlled study in which 48 subjects were enrolled. Thirty-two healthy subjects were randomized in a 3:1 ratio (erenumab:placebo) to receive erenumab SG 21, 70, 140, $210 \mathrm{mg}$ x 3 doses, 280 x 1 dose $+210 \mathrm{mg}$ x 2 doses, or 3 doses of placebo; 16 migraine subjects were randomized in a 3:1 ratio (erenumab:placebo) to receive erenumab SC 21 or 140 mg x 3 doses. Each erenumab or placebo dose was administered every 4 weeks (Q4W). Sampling schedules for collections of PK and DBF measurements are shown in Table I. In both the SAD and MAD studies, erenumab was administered to all subjects (healthy subjects and migraine patients) at protocol-specified visits regardless of the timing of migraine attacks [12, 13].

Studies were conducted at a single center in Leuven, Belgium, approved by the independent ethics committee affiliated with the study center, and performed in accordance with the Declaration of Helsinki. All subjects provided written informed consent. 
Table I Summary of Pharmacokinetic and Pharmacodynamic Studies

\begin{tabular}{|c|c|c|c|c|}
\hline Study code & Dose cohorts (mg) & Number of subjects & PK sampling times & PD sampling times \\
\hline Single ascending dose & & 60 & & \\
\hline Healthy subjects & $\begin{array}{l}\text { Placebo, I, 7, 21, 70, I40, } \\
\text { or } 210 \mathrm{mg} \mathrm{SC} ; 140 \mathrm{mg} \mathrm{IV}\end{array}$ & 48 & \multirow{3}{*}{$\begin{array}{l}\text { Days I (pre-dose and } 0.5^{\mathrm{a}} \text {, } \\
\text { I }^{\mathrm{a}} \text {, and } 8 \mathrm{~h} \text { post-dose), } 2-5, \\
8,12,15,22,29,43,57, \\
64,85,99 \text {, and } 127\end{array}$} & \multirow{3}{*}{$\begin{array}{l}\text { At screening (days }-21 \text { to }-2^{b} \text {, } \\
\text { and day }-1 \text { ) and days } 2^{c}, 4^{c}, 15 \\
29,43,64,85,99,127 \text {, and } 155\end{array}$} \\
\hline Migraine patients & Placebo or $140 \mathrm{mg} \mathrm{SC}$ & 12 & & \\
\hline Multiple ascending dose & & 48 & & \\
\hline Healthy subjects & $\begin{array}{l}\text { Placebo, 2I, 70, I40 mg SC; } \\
280 \mathrm{mg} \times 1+210 \mathrm{mg} \times 2 \mathrm{SC}\end{array}$ & 32 & \multirow{3}{*}{$\begin{array}{l}\text { Days I (pre-dose and } 8 \text { h post-dose), } \\
\text { 4, 5, 8, I2, I5, 22, } 29 \text { (pre-dose), } \\
36,57^{d} \text { (pre-dose and } 8 \text { h post-dose), } \\
64,71,85,99,113,127,169^{d}, 197^{d} \\
\text { and } 224\end{array}$} & \multirow{3}{*}{$\begin{array}{l}\text { At screening (days }-35 \text { to }-2 \text { ) } \\
\text { and days }-1,8,57 \text { (pre-dose), } \\
85,113,169 \text {, and } 197\end{array}$} \\
\hline & $\mathrm{Q} 4 \mathrm{~W} \times 3$ doses & & & \\
\hline Migraine patients & $\begin{array}{l}\text { Placebo, } 21 \text {, or } 140 \mathrm{mg} \mathrm{SC} \\
\mathrm{Q} 4 \mathrm{~W} \times 3 \text { doses }\end{array}$ & 16 & & \\
\hline
\end{tabular}

${ }^{\text {a }} \mathrm{PK}$ sample collection time points required for IV cohort only

${ }^{b}$ For IV cohort, screening DBF performed between days -21 to -4

${ }^{\mathrm{C}} \mathrm{DBF}$ performed on day 2 for IV cohort and on day 4 for SC cohort

${ }^{\mathrm{d}} \mathrm{PK}$ sample collection coincided with dermal blood flow measurement

DBF Dermal blood flow, IV Intravenous, PD Pharmacodynamic, PK Pharmacokinetic, Q4W Every 4 weeks, SC Subcutaneous

\section{Bioanalytical Method}

Erenumab in human serum was quantified according to the validated analytical procedure for the quantification of erenumab in human serum that was developed at Amgen Inc. (Thousand Oaks, CA, USA). Standards (STDs) and quality controls (QCs) were prepared by spiking erenumab into $100 \%$ human serum. STD, QC, blank, and study samples were added to a plate that had been passively coated with a mouse anti-erenumab monoclonal antibody (mAb). After capture of erenumab to the immobilized antibody, unbound materials were removed by a wash step. Biotin-conjugated mouse anti-erenumab $\mathrm{mAb}$ was added for detection of captured erenumab. After another incubation step with streptavidin-HRP, a tetramethylbenzidine peroxide substrate solution was added to produce a colorimetric signal that was proportional to the amount of erenumab bound by the capture reagent. The color development was stopped by addition of sulfuric acid, and the instrument response was measured at $450 \mathrm{~nm}$ with reference to $650 \mathrm{~nm}$. The instrument response vs concentration relationship was regressed according to a fourparameter logistic (Marquardt) regression model with a weighting factor of $1 / \mathrm{Y}^{2}$. The conversion of instrument response for QCs and study samples to concentrations was performed using Watson LIMS (v7.4; Thermo, PA, USA) data reduction software. The lower and upper limits of quantification were $1.00 \mathrm{ng} / \mathrm{mL}$ and $100.00 \mathrm{ng} / \mathrm{mL}$, respectively.

\section{Dermal Blood Flow Measurements}

In both clinical studies, the CIDBF PD assay as described by Van der Schueren et al. [5] was used to determine GGRP receptor antagonism. Briefly, subjects received topical doses of $1000 \mu \mathrm{g}$ capsaicin per $20 \mu \mathrm{L}$ vehicle (i.e., a 3:3:4 mixture of ethanol 100\%, Tween-20, and distilled water). Capsaicin was applied at two sites on the volar surface of a subject's left or right forearm, and vehicle only was applied to one site on the volar surface of the same arm as a control. DBF was assessed by laser Doppler perfusion imaging (Periscan PIM III, Perimed AB, Sweden) and was done immediately before (i.e., "baseline" perfusion) and $0.5 \mathrm{~h}$ after capsaicin application on the skin at these three sites. DBF measurements were performed before erenumab dosing (i.e., pre-dose) and at prespecified time points after dosing as outlined in Table I.

\section{PK and PD Model Development}

\section{PK Model}

The schematic for the PK-PD model is shown in Supplementary Fig. S1. A two-compartment TMDD model was used to describe the PK of erenumab after SC and IV administrations. SC absorption of erenumab was described with first-order absorption rate. Unbound erenumab concentrations were eliminated from the central compartment via a linear elimination pathway and a saturable elimination pathway attributed to the target-mediated mechanism that was described by a Qss model [14]. The equations used to describe the PK system were as follows:

$$
\begin{aligned}
& \frac{d A_{S C}}{d t}=-k_{a} \cdot A_{S C} \\
& A_{S C}(0)=\frac{1}{1+\exp (F)} \cdot \text { Dose }_{S C}
\end{aligned}
$$

where $\mathrm{F}$ estimate is from negative infinity to positive infinity and the ratio $1 /(1+\exp (\mathrm{F}))$ is the total fraction of bioavailable 
dose; $\mathrm{k}_{\mathrm{a}}$ is the first-order absorption rate constant; $\mathrm{A}_{\mathrm{SC}}$ is the amount absorbed; and Dose $_{\mathrm{SC}}$ is the SC dose administered at time $t=0$. The Qss constant $\left(\mathrm{K}_{\mathrm{ss}}\right)$ was estimated directly from the available unbound serum erenumab concentration data, which is derived as:

$$
\begin{aligned}
& {[A M G 334]_{\text {unbound }}=} \\
& 0.5 \cdot\left[\left(\frac{\mathrm{A}_{t o t}}{V_{c}}-R_{t o t}(t)-K_{s s}\right)+\sqrt{\left(\frac{A_{t o t}}{V_{c}}-R_{t o t}(t)-K_{s s}\right)^{2}+4 \cdot K_{s s} \cdot \frac{A_{t o t}}{V_{c}}}\right]
\end{aligned}
$$

Where $\mathrm{R}_{\text {tot }}(\mathrm{t})$ is determined using differential equation $\frac{d R_{t o t}}{d t}=k_{s y n}-k_{\mathrm{deg}} \cdot R_{t o t}-\left(k_{i n t}-k_{\mathrm{deg}}\right) \cdot\left(\frac{A_{t o t}}{V_{c}}-[\mathrm{AMG} 334]_{\text {unbound }}\right)$, with initial condition $\mathrm{R}_{\mathrm{tot}}(0)=\mathrm{k}_{\mathrm{syn}} / \mathrm{k}_{\mathrm{deg}}, \mathrm{k}_{\mathrm{syn}}$ is the zero-order receptor production rate and $\mathrm{k}_{\mathrm{deg}}$ is the first-order receptor degradation rate. The total erenumab amount in the central compartment $\left(\mathrm{A}_{\text {tot }}\right)$ and unbound erenumab amount in the peripheral compartment $\left(A_{p}\right)$ are estimated using differential equations as follows:

$$
\begin{aligned}
\frac{d A_{t o t}}{d t} & =k_{a} \cdot A_{S C}+k_{p c} \cdot A_{p}-k_{\text {int }} \cdot A_{\text {tot }} \\
& -\left(k_{c p}+k_{c 0}-k_{\text {int }}\right) \cdot[\mathrm{AMG} 334]_{\text {unbound }} \cdot V_{c}
\end{aligned}
$$

$$
\frac{d A_{p}}{d t}=k_{c p} \cdot[\mathrm{AMG} 334]_{\text {unbound }} \cdot V_{c}-k_{p c} \cdot A_{p}
$$

where the initial conditions are $\mathrm{A}_{\mathrm{tot}}(0)=\operatorname{Dose}_{\mathrm{IV}}$ and $\mathrm{A}_{\mathrm{p}}(0)=0$; $\mathrm{V}_{\mathrm{c}}$ is the volume of distribution in the central compartment; $\mathrm{k}_{\text {int }}$ is the internalization rate constant for bound erenumab; and $\mathrm{k}_{\mathrm{pc}}, \mathrm{k}_{\mathrm{cp}}$, and $\mathrm{k}_{\mathrm{c} 0}$ are the peripheral to central compartment, central to peripheral compartment, and linear elimination rate constants, respectively.

The total systemic clearance for erenumab was computed with Eq. 5, where $\mathrm{CL}_{\text {linear }}$ is a parameter estimated by the PK model and $\mathrm{CL}_{\text {nonlinear }}$ is a parameter derived from a combination of target-mediated parameters $\left(\mathrm{R}_{\mathrm{tot}, \mathrm{ss}}, \mathrm{K}_{\mathrm{ss}}\right.$, and $\left.\mathrm{k}_{\mathrm{int}}\right)$ estimated by the PK model. $\mathrm{CL}_{\text {nonlinear }}$ is derived using Eq. 6 under the Qss assumption [14, 15].

$$
\begin{aligned}
& C L_{\text {total }}=C L_{\text {linear }}+C L_{\text {nonlinear }} \\
& C L_{\text {nonlinear }}=\frac{k_{\text {int }}}{K_{s s}} \cdot V_{c} \cdot R_{\text {tot }, s s} \cdot\left(1-\frac{A M G 334}{A M G 334+K_{s s}}\right)
\end{aligned}
$$

\section{PD Model}

Individual PK parameters from the PK model were used to generate concentrations as predictor of $\mathrm{CIDBF}$. The response variable was $\triangle \mathrm{DBF}_{\mathrm{t}}$, which was computed as the difference of DBF measurements after and before capsaicin challenge at time t (e.g., day after first dose). After correcting for baseline $\triangle \mathrm{DBF}$ on day 0 , the erenumab effect on DBF after capsaicin challenge was estimated. Serum concentration of erenumab in the central compartment was either indirectly or directly linked to the DBF changes over time, proportional to baseline $\mathrm{DBF}$ on day $0\left(\triangle \mathrm{DBF}_{0}\right)$ as follows:

$\Delta D B F_{t}=\Delta D B F_{0} \cdot\left(1-\frac{I_{\max } \cdot[\mathrm{AMG} 334]^{\text {hill }}}{I C_{50} 0^{\text {hill }}+[\mathrm{AMG} 334]^{\text {hill }}}\right)$

where $I_{\max }$ is the maximum inhibition of DBF due to erenumab treatment, $\mathrm{IC}_{50}$ is a potency parameter or estimate of serum erenumab concentration at $50 \%$ of $\mathrm{I}_{\max }$ and erenumab is the serum concentration in the central compartment. To assess the threshold (or on/off) effect of erenumab on DBF, a sigmoidicity parameter or hill coefficient was also estimated. To estimate the erenumab concentration needed to achieve maximum possible DBF inhibition, $\mathrm{IC}_{99}$ was derived as $99^{1 / \text { hill }} \cdot \mathrm{IC}_{50}$.

The observed erenumab inhibitory effect on capsaicininduced change in DBF was reported as the percent change in $\triangle \mathrm{DBF}_{\mathrm{t}}$ relative to $\triangle \mathrm{DBF}_{0}$, i.e., inhibition $(\%)=100$. $\left(\triangle \mathrm{DBF}_{\mathrm{t}} / \triangle \mathrm{DBF}_{0}-1\right)$. The model predicted erenumab inhibition was defined by the $\mathrm{I}_{\max } \cdot[\text { erenumab }]^{\text {hill }} /\left(\mathrm{IC}_{50}\right.$ hill + [erenumab] ${ }^{\text {hill }}$ ) term in Eq. 7 above.

Details of additional methods including statistical model, covariate analyses, model evaluation criteria, simulations, and software used are provided as supplemental material (see Supplementary Methods).

\section{RESULTS}

\section{Subjects and Data}

The pooled analysis dataset had PK-PD data for 108 subjects: 60 in the SAD study and 48 in the MAD study. Erenumab concentration data were available for 78 subjects and repeated-measurements of $\mathrm{DBF}$ data were available for 100 subjects, 30 of whom received placebo.

Of the 108 subjects, $80(74 \%)$ were healthy subjects and $28(26 \%)$ were migraine patients; 78\% were male. The mean (standard deviation, SD) age of all subjects was 29.5 (9.7) years and the mean (SD) weight was 74.9 (11.9) $\mathrm{kg}$ (Table II).

A total of 1297 erenumab serum concentrations from 78 subjects were available for population PK analysis, and 676 DBF measurements from 100 subjects were available for population PK-PD analysis. Of these samples, 82 (6\%) PK 
Table II Characteristics of Treated Subjects Included in the Pharmacokinetic-Pharmacodynamic Analysis

\begin{tabular}{|c|c|c|c|}
\hline Parameter & Single-dose study & Multiple-dose study & All \\
\hline n (\%) & $N=60$ & $N=48$ & $N=108$ \\
\hline \multicolumn{4}{|l|}{ Sex } \\
\hline Male & $51(85)$ & $33(69)$ & $84(78)$ \\
\hline Female & $9(15)$ & $15(3 \mid)$ & $24(22)$ \\
\hline \multicolumn{4}{|l|}{ Study subjects } \\
\hline Healthy subjects & $48(80)$ & $32(67)$ & $80(74)$ \\
\hline Migraine patients & $12(20)$ & $16(33)$ & $28(26)$ \\
\hline \multicolumn{4}{|l|}{ SC dose groups } \\
\hline Erenumab & $36(60)$ & $36(75)$ & $72(67)$ \\
\hline Placebo & $16(27)$ & $12(25)$ & $28(26)$ \\
\hline \multicolumn{4}{|l|}{ IV dose groups } \\
\hline Erenumab & $6(10)$ & - & $6(5)$ \\
\hline Placebo & $2(3)$ & - & $2(2)$ \\
\hline \multicolumn{4}{|l|}{ Mean \pm SD } \\
\hline Age (years) & $27.2 \pm 7.3$ & $32.4 \pm 11.4$ & $29.5 \pm 9.7$ \\
\hline Male & $27.0 \pm 6.8$ & $31.4 \pm 10.1$ & - \\
\hline Female & $28.1 \pm 10.4$ & $34.5 \pm 14.0$ & - \\
\hline Body weight, (kg) & $76.7 \pm 11.1$ & $72.7 \pm 12.7$ & $74.9 \pm 11.9$ \\
\hline Male & $79.2 \pm 9.3$ & $76.1 \pm 12.4$ & - \\
\hline Female & $62.2 \pm 9.8$ & $65.3 \pm 10.1$ & - \\
\hline
\end{tabular}

$\mathrm{N}=$ number of subjects evaluated in each study; $\mathrm{n}=$ number of subjects for each parameter

IV Intravenous, SC Subcutaneous, SD Standard deviation

measurements below the limit of quantification were excluded from the analysis. A total of 6 subjects developed binding antierenumab antibodies during the studies $(1$ in $\mathrm{SAD}$ and 5 in MAD); 1 subject (in the MAD study) was positive for neutralizing antibodies. The effect of anti-erenumab binding antibodies on PK parameters was not assessed due to a small number of observations.

\section{Pharmacokinetic Model}

The population parameter estimates from a final twocompartment TMDD model with first-order absorption for SC administration is shown in Table III. To avoid model over-parameterization, the quasi-steady-state (Qss) model under the Qss assumption was used as an approximation of the general two-compartment TMDD model [14, 16]. The target receptor concentration was estimated to change over time.

The linear non-specific clearance was estimated to be $0.214 \mathrm{~L} /$ day (between subject variability [BSV]: $25 \%$ ) and the central volume of distribution was $4.27 \mathrm{~L}$ (BSV: $44 \%$ ). The rate constant of the complex internalization $\left(\mathrm{k}_{\text {int }}: 0.0345\right.$ day $\left.^{-1}\right)$ was similar to the linear elimination of free erenumab $\left(\mathrm{CL} / \mathrm{V}_{\mathrm{c}}\right.$ : 0.0501 day $^{-1}$ ). Maximal nonlinear clearance, derived from $\mathrm{k}_{\text {int }} \cdot \mathrm{V}_{\mathrm{c}} \cdot \mathrm{R}_{\mathrm{tot}, \mathrm{ss}} / \mathrm{K}_{\mathrm{ss}}$, was approximately $1.84 \mathrm{~L} /$ day and 8 times the linear clearance (Supplementary Fig. S2).

The precision of fixed- and random-effect parameter estimates was acceptable. The largest BSV estimate was in the absorption rate $\mathrm{k}_{\mathrm{a}}(60 \%)$. Residual variability in erenumab concentrations was low-approximately $18 \%$ of the predicted concentrations, with a reasonable shrinkage of $11 \%$. Correlations were observed in the random effects for PK parameters, -0.49 between $\mathrm{k}_{\mathrm{a}}$ and CL, and 0.642 between CL and $k_{\text {int }}$.

Population predictions were significantly improved for the best-fitted model with $\mathrm{CL}$ and $\mathrm{V}_{\mathrm{c}}$ adjusted for body weight $(p<0.001)$. The exponents of the effect of body weight on CL and $\mathrm{V}_{\mathrm{c}}$ were fixed to allometric exponents of 0.75 and 1 , respectively. Model fitting was not significantly better when estimating the exponents than when fixing them. When estimated, the exponents were 0.532 and 1.64 for $C L$ and $V_{c}$, respectively, and the change in minimum value of objective function was not statistically significant when comparing to the model with fixed allometric exponents $(p>0.001)$. Since all migraine patients were female, evaluation of sex covariate was the same as that of population (e.g., migraine vs. healthy). Sex and age, after adjusting for body weight, did not show statistical significances at $p<0.001$ on $\mathrm{CL}$ or $\mathrm{V}_{\mathrm{c}}$ as covariates when estimated. Consistent with observed data, erenumab serum concentration time courses were similar between healthy subjects and migraine patients (Supplementary Fig. S3). Fig. 1 shows the approximately linear relationships between body weight and the individual PK parameters, linear clearance and central volume of distribution. Goodnessof-fit plots (observed vs population and individual predicted concentrations, and residual vs time) did not show systematic bias in model predictions (Supplementary Figs. S4 and S5). The visual predictive check plots as an internal model evaluation showed that the distributions of observed and predicted concentrations were mostly in agreement (Fig. 2), except for lower doses (1 and $7 \mathrm{mg}$ ) because of the small sample size $(\mathcal{N}=3$ for each dose); uncertainty in predictions for the nonlinear concentration range $\left(<\mathrm{R}_{\text {tot }}\right)$ was seen at higher doses, which reflects the lack of $\mathrm{PK}$ information by study design. Nevertheless, prediction corrected visual predictive check (VPC) clearly supported the appropriateness of the PK model after removing the variability largely due to limited data at lower doses/concentrations (Supplementary Fig. S6). Overall, the model captured population PK characteristics and variability across a wide range of doses and in both healthy and migraine populations.

\section{Pharmacodynamic Models}

A simple inhibitory $\mathrm{E}_{\max }$ model was used to describe the effect of erenumab on the time course of DBF. Erenumab is assumed to have no effect on DBF over time in the 
Table III Pharmacokinetic Parameter Estimates

\begin{tabular}{|c|c|c|c|c|}
\hline Parameters & Units & Mean estimate & Bootstrapped estimate $(95 \% \mathrm{Cl})$ & Shrinkage (\%) \\
\hline Nonspecific linear clearance $(C L)^{a}$ & L/day & 0.214 & $0.213(0.191,0.243)$ & \\
\hline Central volume of distribution $\left(V_{c}\right)^{a}$ & $L$ & 4.27 & $4.2(3.4,5.38)$ & \\
\hline Intercompartmental clearance (Q) & L/day & 3.34 & $3.25(1.41,6.79)$ & \\
\hline Peripheral volume $\left(V_{p}\right)$ & L & 2.73 & $2.75(2.2,3.18)$ & \\
\hline Absorption rate $\left(k_{a}\right)$ & I/day & 0.432 & $0.428(0.346,0.519)$ & \\
\hline Bioavailability (F) & $\%$ & 74 & $74(66,85)$ & \\
\hline Receptor production rate $\left(\mathrm{k}_{\text {syn }}\right)$ & $\mathrm{ng} / \mathrm{mL} /$ day & 51.9 & $53(40.6,65.4)$ & \\
\hline Receptor degradation rate $\left(k_{\text {deg }}\right)$ & I/day & 0.22 & $0.222(0.144,0.427)$ & \\
\hline Binding affinity $\left(K_{\mathrm{ss}}\right)$ & $\mathrm{ng} / \mathrm{mL}$ & 18.8 & I8.| $(10.6,30.6)$ & \\
\hline Internalization rate $\left(k_{\text {int }}\right)$ & I/day & 0.0345 & $0.0347(0.0251,0.0442)$ & \\
\hline $\mathrm{BSV}$ in $\mathrm{CL}$ & $\% C V$ & 25.1 & $25(21,29.6)$ & 4.3 \\
\hline$B S V$ in $V_{c}$ & $\% C V$ & 43.8 & $43.8(35.3,53)$ & 5.6 \\
\hline BSV in $\mathrm{k}_{\mathrm{a}}$ & $\% C V$ & 59.5 & $58.8(45.6,73.3)$ & 7.9 \\
\hline BSV in $k_{\text {int }}$ & $\% C V$ & 42.3 & $42.1(33.5,51.7)$ & 6.8 \\
\hline Covariance between $\eta C L$ and $\eta k_{a}$ & - & -0.0731 & $-0.0719(-0.127,-0.037 \mid)$ & \\
\hline Covariance between $\eta C L$ and $\eta k_{\text {int }}$ & - & 0.0682 & $0.0664(0.0396,0.101)$ & \\
\hline Residual variability & $\% C V$ & 17.9 & $17.6(15.9,19.7)$ & 10.7 \\
\hline
\end{tabular}

$95 \% \mathrm{Cl}=95 \%$ confidence interval from 1000 bootstrapped runs of the final model

${ }^{a}$ Mean $\mathrm{CL}$ and $\mathrm{V}_{c}$ estimates at $70 \mathrm{~kg}$; individual body weight effect on $\mathrm{CL}$ and $\mathrm{V}_{c}$ were estimated as Individual $\mathrm{CL}=0.2 \mathrm{I} 4$ (weight/70) ${ }^{0.75} \mathrm{~L} / \mathrm{day}$ and Individual $V_{c}=4.27$ (weight/70) L

BSV Between-subject variability expressed as \%CV, CV Approximates coefficient of variation

absence of capsaicin challenge (e.g., at 0 min) but decreases DBF after capsaicin challenge (e.g., at $30 \mathrm{~min}$ ). This is consistent with erenumab's mechanism of action; it attenuates the action of CGRP in the periphery. The time courses of DBF inhibition were similar between healthy subjects and migraine patients (Supplementary Fig. S3). The assumption was verified by the observed data as shown in Supplementary Fig. S7, where DBF before capsaicin challenge did not change as erenumab concentration increased over time. The PD model adequately described the observed DBF time course in both single- and multiple-dose studies (Fig. 3). Prediction-corrected VPC further supported the appropriateness of the PD model to describe DBF time courses across a wide range of doses in phase 1 studies (Supplementary Fig. S6). After adjusting for body weight effect on PK, body weight had no additional effect on PD parameters. Deterministic simulations showed that the DBF inhibition is at maximum for the entire dosing interval at steady-state for the low (25th percentile) and high (75th percentile) body weights, after repeated dosing of $21 \mathrm{mg}$ and 70 mg SC Q4W (Supplementary Fig. S8). All final PD parameter estimates were estimated with good precision (Table IV). Capsaicin challenge increased DBF on average by 398 (95\% CI: 369-428) perfusion units (PU) with a BSV of $28 \% \mathrm{CV}$ in the absence of erenumab; i.e., on average, DBF post-capsaicin challenge (at $30 \mathrm{~min}$ ) increased approximately 10- to 12-fold above DBF pre-capsaicin challenge (at $0 \mathrm{~min}$ ) in the absence of erenumab. The concentration-response curve was sigmoidal and plateaued at approximately $1134 \mathrm{ng} / \mathrm{mL}\left(\mathrm{IC}_{99}\right.$; Fig. 4). Change in $\mathrm{DBF}$ response is highly variable across concentration ranges and $\mathrm{BSV}$ in $\mathrm{IC}_{50}$ is large (\%CV: 94, 95\% CI: 68-120\%). The hill coefficient was estimated to be 3.08 (95\% CI: 0.555-5.61). Maximum inhibition of DBF corrected for baseline was approximately $89 \%$ with good precision $(95 \%$ CI: $87-91 \%$ ).

\section{Model-Based Simulations}

Model-based simulation was performed to support dose selection for a migraine prevention study in subjects with episodic migraine. PK-PD simulations for dose selection was based on the assumptions that DBF inhibition was predictive of migraine efficacy (e.g., reduction of monthly migraine days) and maximum DBF inhibition was required for efficacy. Figure 5 shows the simulation results for 3 selected dose levels of erenumab at monthly dosing intervals. Based on the PK-DBF relationship, $21 \mathrm{mg}$ SC every 4 weeks $(\mathrm{Q} 4 \mathrm{~W})$ is expected to place more than $50 \%$ of subjects with concentrations above $\mathrm{IC}_{99}$ (e.g., concentration at $99 \%$ of maximum DBF inhibition) after the second dose. This regimen is projected to be the minimum migraine efficacious dose. At 

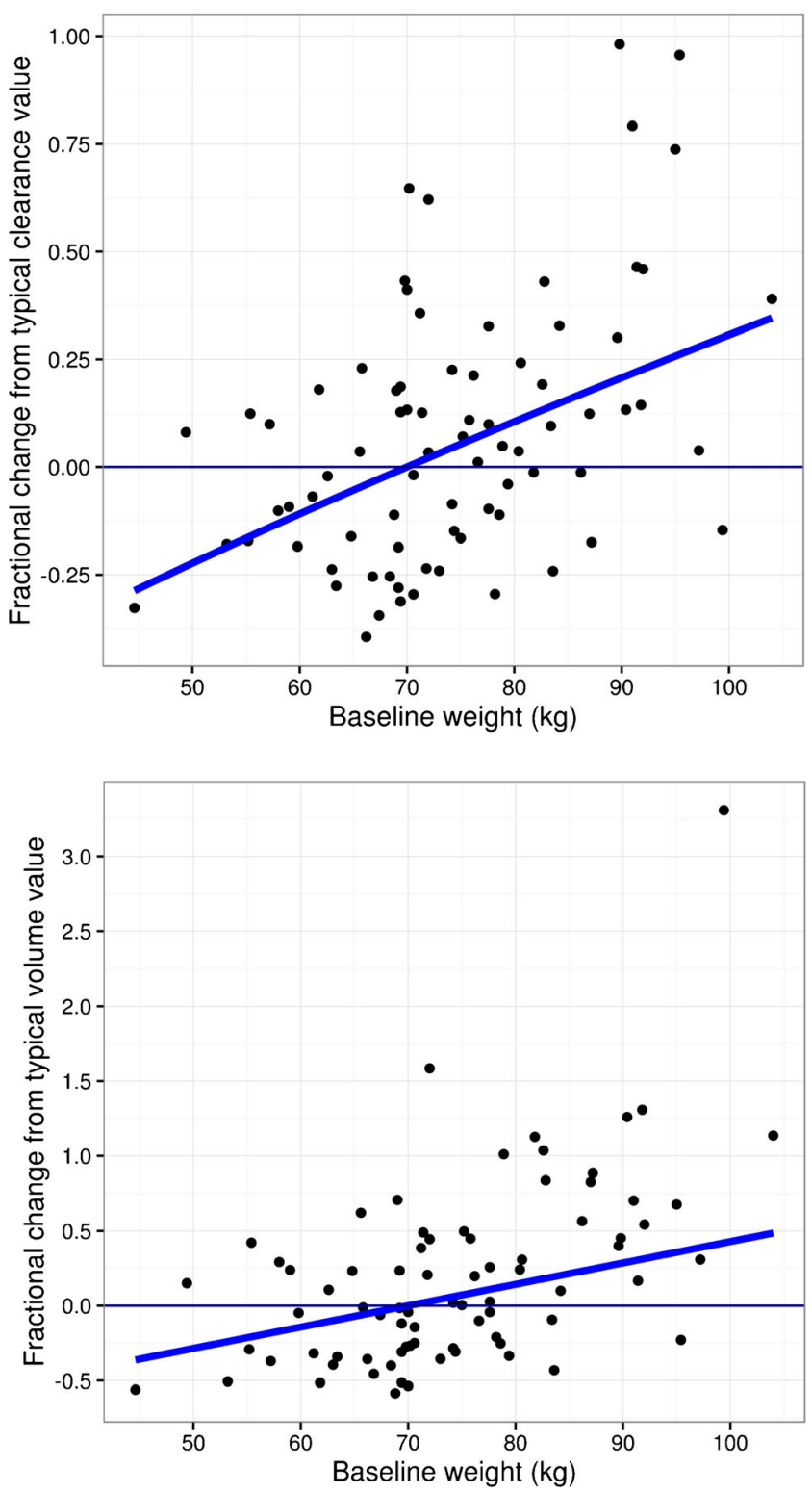

Fig. I Relationship between body weight and pharmacokinetic parameters. Solid lines are typical population trends between parameter estimates and body weight; solid symbols are individual parameter estimates as function of body weight.

$70 \mathrm{mg}$ SC Q4W, all subjects are expected to be above the mean $\mathrm{IC}_{99}$ within the first dose and this dose regimen is projected to have higher migraine efficacy than $21 \mathrm{mg}$. Lastly, $7 \mathrm{mg}$ SC Q4W would provide the migraine efficacy information below the mean $\mathrm{IC}_{50}$, where $50 \%$ of $\mathrm{DBF}$ inhibition is achieved. The percentage of DBF inhibition relative to maximum inhibition provides further information about variability and duration of response (Fig. 5, right panel). At $70 \mathrm{mg} \mathrm{SC}$ Q4W, $100 \%$ of the maximum DBF inhibition had already been achieved for the entire duration of the study. Therefore, migraine efficacy (or reduction of migraine days) was expected to be maintained during the dosing interval.

\section{DISCUSSION}

We have described the PK of erenumab in healthy subjects and migraine patients using a mechanism-based TMDD model under the Qss assumption. The model successfully explained the dose/concentration-dependent elimination of erenumab, which was assumed to be mediated by elimination of the erenumab-receptor complex. We also characterized the PD effect of erenumab by linking the time course of erenumab serum concentrations to the time course of the inhibition of CIDBF.

According to model estimations, the mean absorption time was approximately 2 days and the estimated absorption halflife was 1.6 days, suggesting SC absorption was complete in approximately 8 days. The estimate of SC bioavailability for erenumab is $74 \%(95 \%$ CI: $66-85 \%)$, which is within the reported range for most $\mathrm{mAb}$ therapeutics $(50-100 \%)$ [17]. Similar to other mAb therapeutics with nonlinear PK, erenumab exhibits TMDD behavior that is typically described by two parallel antibody elimination pathways: a slow nonspecific elimination pathway through the hepatic reticuloendothelial system (i.e., linear clearance), and a rapid saturable elimination pathway (i.e., nonlinear clearance) mediated by degradation or internalization of the erenumab-receptor complex. The population estimate of linear clearance is independent of erenumab concentrations and stays approximately constant at $0.214 \mathrm{~L} /$ day $(95 \%$ CI: $0.191-0.243)$, which is very similar to the typical clearance of endogenous $\operatorname{IgG}(\sim 0.21 \mathrm{~L} /$ day) [18], with a low BSV $(21 \% \mathrm{CV})$. In contrast, nonlinear clearance is dependent on the target receptor density and the amount of erenumab bound to the receptors. As shown in Supplemental Fig. S2, total clearance of erenumab decreased significantly and approached linear clearance as erenumab concentrations increased. Consequently, it is expected that dose regimens $\geq 70 \mathrm{mg} \mathrm{SC}$ Q $4 \mathrm{~W}$ would produce concentrations that saturate the nonlinear elimination pathway at steady state (Supplementary Fig. S2).

Body weight is a common covariate found to explain variability in the PK parameters of mAbs [18]. In the current dataset with a body weight range of 49-104 kg, for every $10 \mathrm{~kg}$ increase in body weight, linear clearance and central volume of distribution increased by $11 \%$ and $14 \%$, respectively. Other covariates such as sex and age were highly correlated with body weight and had no further contribution to the variability in PK parameters. The majority of migraine patients were female (21 of 28 with $\mathrm{PK}$ ) and their PK parameters were not significantly different from those of healthy subjects.

Erenumab effectively inhibited CIDBF increase in both healthy subjects and migraine patients at doses of $7 \mathrm{mg}$ or greater (Fig. 3). The relationship between erenumab serum concentrations and DBF inhibition was sigmoidal and reached a plateau at approximately $1134 \mathrm{ng} / \mathrm{mL}$ (Fig. 4). The erenumab concentration-DBF relationship is robust and 

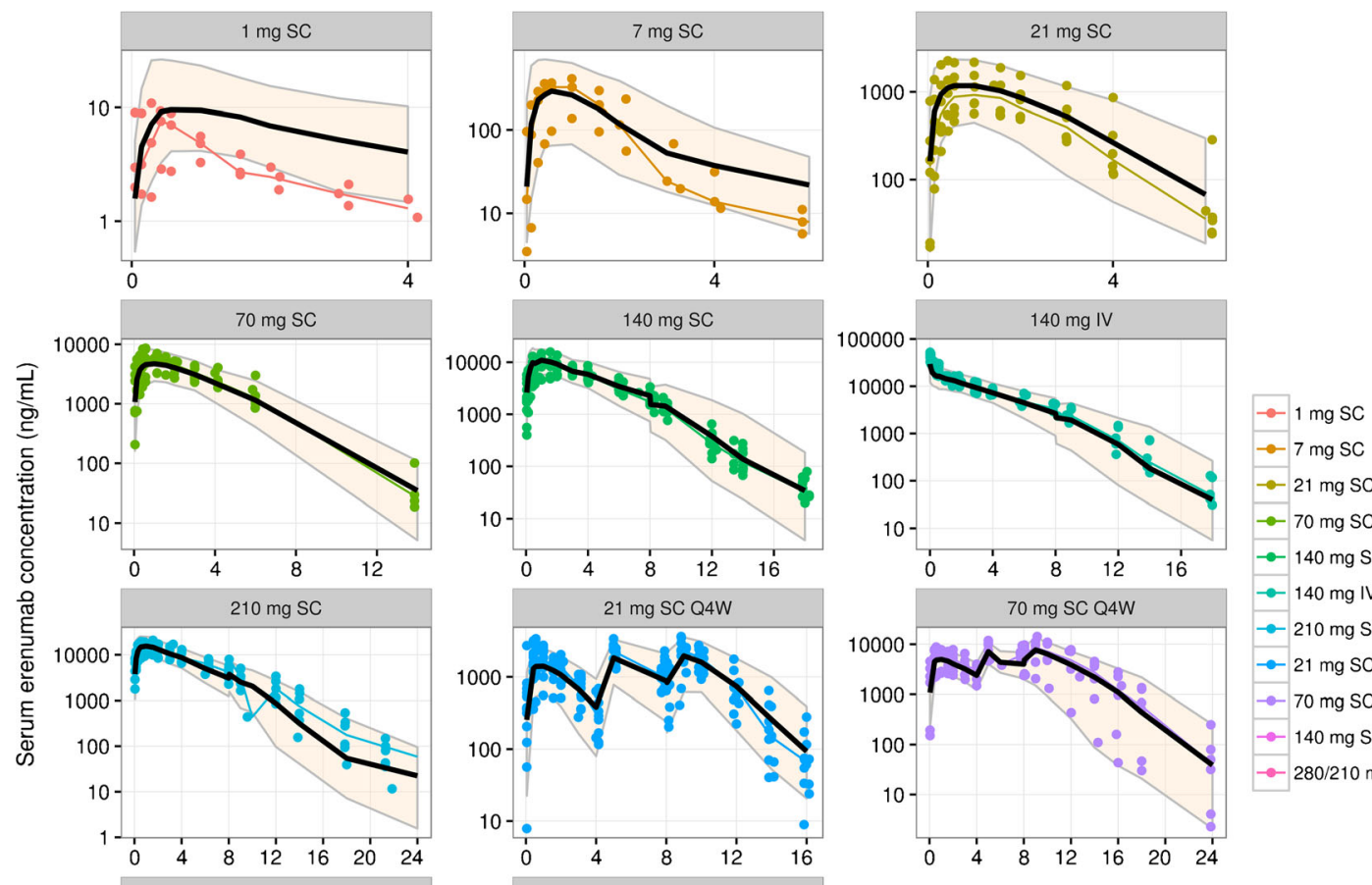

$\rightarrow 21 \mathrm{mg} \mathrm{SC}$

- $70 \mathrm{mg} \mathrm{SC}$

$\rightarrow 140 \mathrm{mg} \mathrm{SC}$
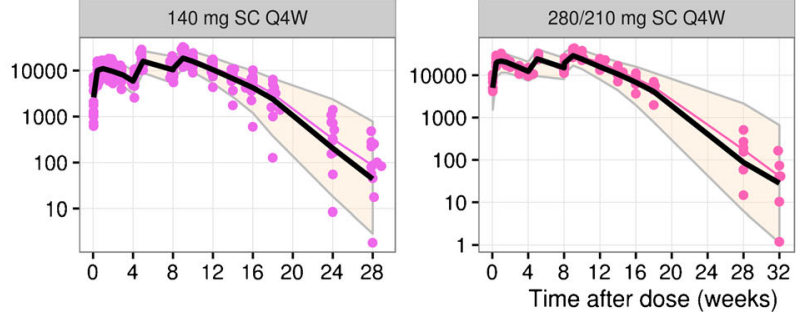

Fig. 2 Visual predictive checks for the final pharmacokinetic model of erenumab concentrations. Colored symbols and lines are individual observations and the medians of the observations for the corresponding dosing cohorts; solid black lines and shaded bands are the model predicted 50th and 90th percentiles. IV, intravenous; Q4W, every 4 weeks; SC, subcutaneous.

maximum inhibition is sustained with repeated dosing, with no evidence of attenuation. An indirect response model did not significantly describe data better than a direct response model, suggesting that erenumab's effect was immediate and there was no evidence of delay in DBF inhibition. Erenumab's concentration effect on DBF inhibition was not significantly different between healthy subjects and migraine patients, indicating that the biomarker model (i.e., CIDBF increase) is translatable from healthy subjects to migraine patients. Although body weight had a significant effect on erenumab serum concentrations, at the clinical doses of interest (i.e., $21 \mathrm{mg}$ and $70 \mathrm{mg}$ SC Q4W), body weight did not have an impact on DBF inhibition since the concentrations were well above the required concentration for maximum DBF inhibition (i.e., $\mathrm{IC}_{99}$, Supplementary Fig. S8).

Erenumab is highly potent in inhibiting capsaicin-induced increases in DBF in humans, with an $\mathrm{IC}_{50}$ of $255 \mathrm{ng} / \mathrm{mL}$ or $1.7 \mathrm{nM}$ estimated with excellent precision $(95 \%$ CI: $115-$ $395 \mathrm{ng} / \mathrm{mL}$ ). Thus, compared with telcagepant, an oral CGRP receptor antagonist with an $\mathrm{IC}_{50}$ of $101 \mathrm{nM}$ [19], erenumab is much more potent. Erenumab has a similar potency to MK-3207 $\left(\mathrm{IC}_{50}=1.59 \mathrm{nM}\right)$, another oral CGRP receptor antagonist [20]. At a minimum, an erenumab dose of $7 \mathrm{mg}$ SC easily provided serum concentrations to achieve target coverage. The maximum possible DBF inhibition estimated by modeling was $89 \%$ (95\% CI: 87.3-91.4), which is comparable with the estimated maximum inhibition of $92 \%$ achieved with telcagepant [19]. These findings are consistent with the fact that, next to CGRP, other bioactive mediators are most likely involved in neurogenic inflammation induced by the activation of TRPV1 receptors in humans. Because previous studies excluded a substantial involvement of vasodilating prostaglandins, nitric oxide, and Substance $\mathrm{P}$, the identities of additional mediators remain to be determined [21].

Compared with mAbs in development that target the CGRP ligand, such as LY2951742 [6], TEV-48125, and ALD-403 [22] erenumab requires a lower concentration to achieve and maintain maximum CIDBF inhibition; and the magnitude of maximum CIDBF inhibition is higher. For example, ALD-403 required approximately $308 \mathrm{nM}$ $\left(\mathrm{IC}_{99}=46,163 \mathrm{ng} / \mathrm{mL}\right)$ to reach a CIDBF inhibition plateau of only $61 \%$. 

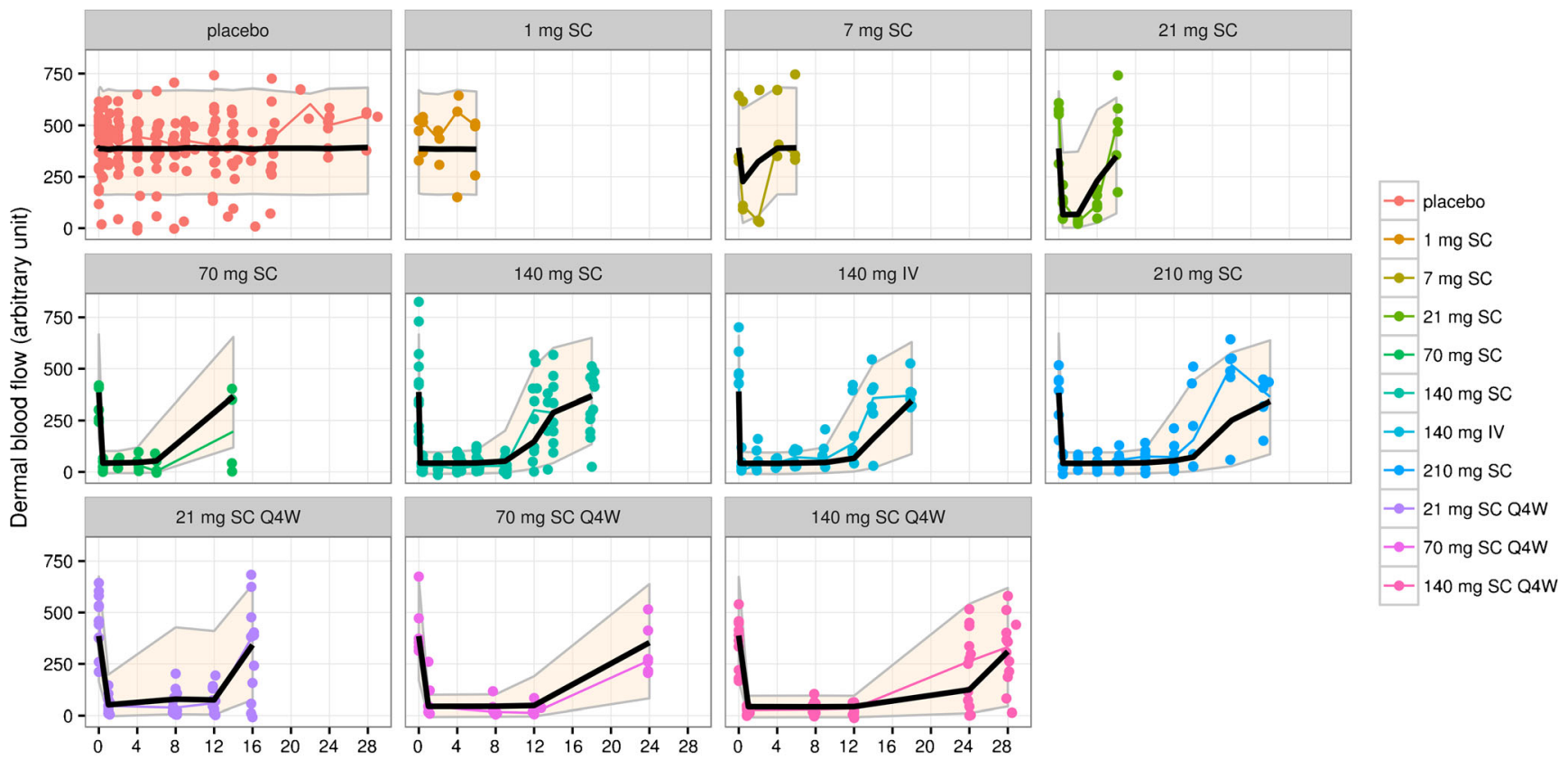

Fig. 3 Visual predictive checks for the final pharmacodynamic model of dermal blood flow for erenumab. Colored symbols and lines are individual observations and the medians of the observations for the corresponding dosing cohorts; solid black lines and shaded bands are the model predicted 50th and 90th percentiles. IV, intravenous; Q4W, every 4 weeks; SC, subcutaneous.

It is important to note that although erenumab potently inhibits the capsaicin-induced increase in DBF, the basal DBF is not affected. Consequently, although CGRP is a potent endogenous vasodilator, it does not seem to be involved in maintaining basal peripheral vascular tone. Likewise, it has also been reported that the small molecule CGRP receptor antagonists olcegepant and telcagepant do not affect resting tissue perfusion. Taken together, these data suggest that CGRP receptor antagonists may be devoid of cardiovascular side-effects under resting conditions.

The major benefit of establishing the relationship between erenumab concentration and DBF inhibition is the ability to predict the erenumab exposures, magnitude, and duration of peripheral target engagement, and their theoretical relationship to efficacy in the target population. This is the cornerstone of model-based drug development that is used to guide dose selection in phase 2 dose ranging studies. We used the erenumab concentration-DBF model to select dose regimens for a dose-ranging study design under the assumption that maximum DBF inhibition is required for migraine efficacy (Fig. 5). Three doses, 7, 21, and $70 \mathrm{mg} \mathrm{SC}$ monthly were selected to provide dose-response information. Based on the simulation results, both $21 \mathrm{mg}$ and $70 \mathrm{mg}$ SG monthly regimens were predicted to be effective doses in the episodic migraine population, with the $70 \mathrm{mg}$ SC monthly regimen predicted to have better efficacy. The $7 \mathrm{mg} \mathrm{SC}$ dose was added to

Table IV Pharmacodynamic Parameter Estimates

\begin{tabular}{|c|c|c|c|}
\hline Parameter & Units & Mean estimate $(95 \% \mathrm{Cl})$ & Shrinkage (\%) \\
\hline Baseline dermal blood flow & PU & $398(369,428)$ & \\
\hline Potency $\left(\mathrm{IC}_{50}\right)$ & $\mathrm{ng} / \mathrm{mL}$ & $255(115,395)$ & \\
\hline Maximum inhibition $\left(I_{\max }\right)$ & $\%$ & $89.3(87.3,91.4)$ & \\
\hline Hill coefficient & & $3.08(0.555,5.61)$ & \\
\hline \multirow[t]{2}{*}{ Residual variability } & PU & $23.8(16.1,31.5)$ & 3.6 \\
\hline & $\% C V$ & $21.9(17.2,26.6)$ & 3.6 \\
\hline BSV in baseline dermal blood flow & $\% C V$ & $28.3(23.3,33.2)$ & 5.4 \\
\hline BSV in $\mathrm{IC}_{50}$ & $\% C V$ & $94.1(68.1,120)$ & 12.2 \\
\hline Residual error & $\% C V$ & $38.7(27.1,50.2)$ & 33.3 \\
\hline
\end{tabular}

$95 \% \mathrm{Cl}=95 \%$ confidence interval as estimated by the Wald's test of mean estimate \pm 1.96 .standard error

BSV Between-subject variability expressed as \%CV, CV Approximates coefficient of variation, PU Perfusion unit 
Fig. 4 Relationship between erenumab concentrations and dermal blood flow (DBF) inhibition. The symbols represent individual observations. IV, intravenous; Q4W, every 4 weeks; SC subcutaneous.

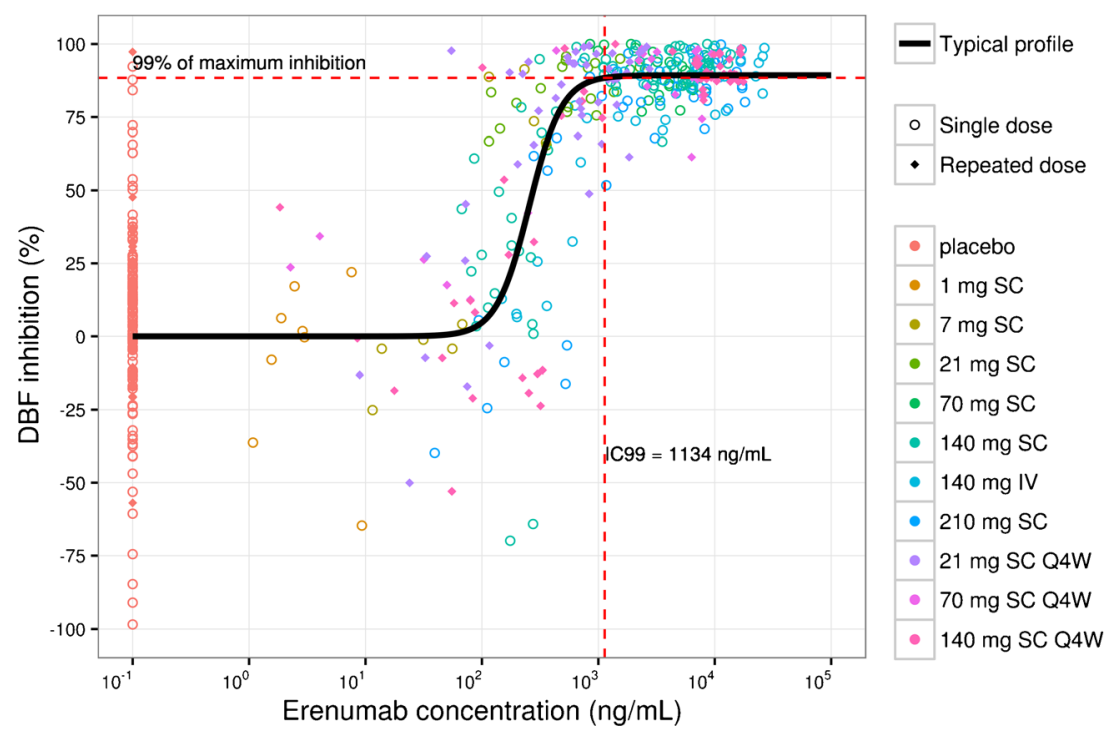

demonstrate a non-effective dose. Since these predictions of migraine efficacy were theoretical we took a conservative approach by selecting $70 \mathrm{mg}$ as the highest dose regimen, which exceeded $\mathrm{IC}_{99}$ by 7 -fold after the first dose and 10 -fold after the third dose. Results from the dose-ranging study in episodic migraine patients demonstrated a separation from placebo for only the $70 \mathrm{mg}$ SC monthly dose (not for lower doses of $7 \mathrm{mg}$ and $21 \mathrm{mg}$ ) [23]. These results indicate that the CIDBF pharmacology model, despite being a well validated translational biomarker to provide evidence of peripheral target engagement, does not necessarily predict exposures needed to obtain anti-migraine efficacy [24].

Interestingly, a comparable observation was reported for telcagepant [19] in that the effective therapeutic doses in acute migraine clinical trials resulted in mean plasma concentrations that were approximately 2 - to 4 -fold higher than the $\mathrm{IC}_{90}$ in the capsaicin model, suggesting that larger doses than those producing maximal peripheral CGRP receptor inhibition are necessary for anti-migraine efficacy. Given these observations, it has been suggested that therapeutic efficacy in the treatment of migraine, traditionally thought to be a central nervous system (CNS) disorder, might require higher doses than expected based on peripheral target engagement. Indeed, if penetration in the CNS is needed, peripheral target engagement might not be representative of concentrations needed to achieve central target engagement, as mAb concentrations in the CNS are only $\sim 0.1 \%$ of their serum concentrations [25]. However, increasing evidence suggests that central penetration is not needed to obtain anti-migraine efficacy. Recent PET data with telcagepant convincingly showed that, at therapeutic doses, no meaningful occupancy of CGRP-receptors was observed in the CNS [26]. This observation supports the concept that, to relieve migraine, it is sufficient for CGRP-blocking therapeutics, including mAbs, to act peripherally. However, this does not exclude the possibility that compounds acting both peripherally and centrally might have added value.

There are some limitations in the current analysis. The TMDD model implied that the erenumab nonlinear elimination pathway is mediated by its binding to the CGRP receptor. However, the density/amount of CGRP receptors in
Fig. 5 Predicted erenumab pharmacokinetics (PK) (left panel) and dermal blood flow (DBF) inhibition (right panel) for the selected doses in the phase 2 trial. Solid lines represent median population predictions; dashed lines represent $90 \%$ prediction intervals that account for total variability in $\mathrm{PK}$ or DBF response. The predicted DBF inhibition is expressed as percentage of the maximum possible inhibition (percent of $\left.I_{\max }[89 \%]\right)$. QM, monthly.
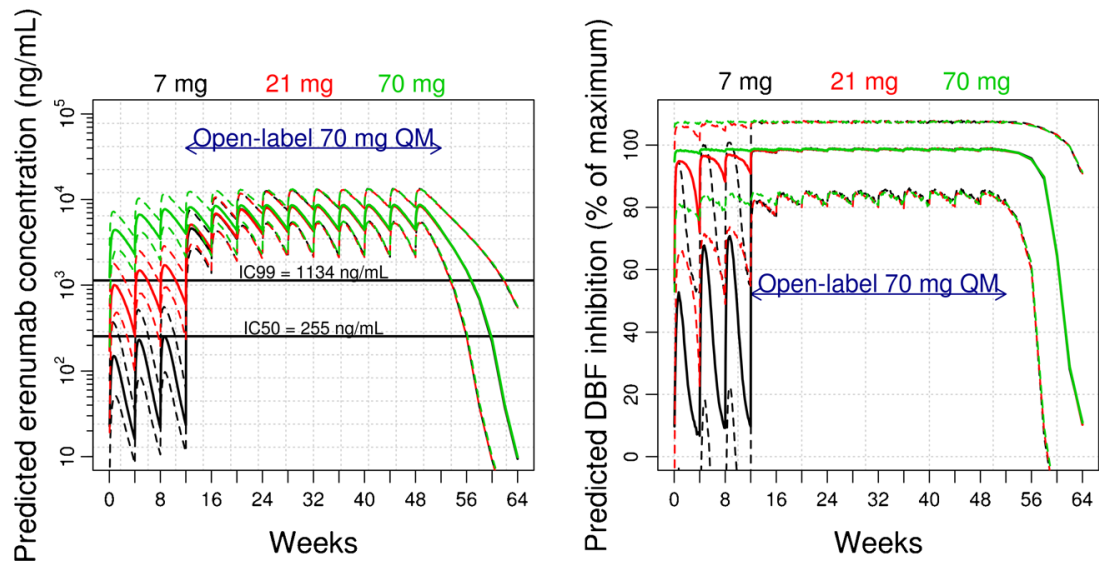
humans is not known; therefore, the PK parameters related to target-mediated elimination are data driven. The covariate effects evaluated in this PK-PD analysis are applicable only to the observed range of baseline characteristics in the included studies. Therefore, covariates that are found not significant in this analysis should be re-evaluated in population analysis where a broader population of subjects are included.

\section{CONCLUSIONS}

The PK characteristics of erenumab are typical of mAb therapeutics that exhibit TMDD. Furthermore, the erenumab concentration-DBF relationship indicates that erenumab is highly potent against peripheral CGRP receptors and that its pharmacological effect is sustained with repeated administration. Erenumab shows maximum target engagement after single and repeated dosing, and has a long serum effective half-life at doses $\geq 70 \mathrm{mg} \mathrm{SC}$. The established PK-PD relationship of erenumab provides a useful tool to select appropriate dose regimens and study design for future clinical studies in migraine patients.

\section{ACKNOWLEDGMENTS AND DISCLOSURES}

This study was funded by Amgen Inc. The authors thank the staff at the Centre for Clinical Pharmacology at University Hospitals of Leuven, especially Jo Van Effen and Marissa Herbots, for assisting in the experiments and data collection. The authors also acknowledge Adimoolam Narayanan of Amgen Inc. for programming support and study summaries. Lisa Humphries of Amgen Inc. and Martha Mutomba (on behalf of Amgen Inc.) provided formatting, editing, and submission support.

TV, LY, LH, and GV are employees of Amgen and may have Amgen stock/stock options; LSW, PM, and JSC were employees of Amgen at the time these analyses were conducted. JdH reports research grants from Abide, Amgen, Galderma, Genentech, GlaxoSmithKline, Janssen Research \& Development, Lilly Chorus, MSD, Novartis, Sanofi Pasteur, UCB, and Vertex and reports consultancy for Ablynx, Amgen, Eli Lilly, Genentech, and UCB. AVH has declared no conflicts of interest.

Open Access This article is distributed under the terms of the Creative Commons Attribution 4.0 International License (http://creativecommons.org/licenses/by/4.0/), which permits unrestricted use, distribution, and reproduction in any medium, provided you give appropriate credit to the original author(s) and the source, provide a link to the Creative Commons license, and indicate if changes were made.

\section{REFERENCES}

1. Lipton RB, Bigal ME, Diamond M, Freitag F, Reed ML, Stewart WF, et al., Group AA. Migraine prevalence, disease burden, and the need for preventive therapy. Neurology. 2007;68(5):343-9.

2. Lipton RB, Stewart WF, Diamond S, Diamond ML, Reed M. Prevalence and burden of migraine in the United States: data from the American migraine study II. Headache. 2001;41(7):646-57.

3. Stewart WF, Lipton RB, Celentano DD, Reed ML. Prevalence of migraine headache in the United States. Relation to age, income, race, and other sociodemographic factors. JAMA. 1992;267(1):64-9.

4. Robbins MS, Lipton RB. The epidemiology of primary headache disorders. Semin Neurol. 2010;30(2):107-19.

5. Van der Schueren BJ, de Hoon JN, Vanmolkot FH, Van Hecken A, Depre M, Kane SA, et al. Reproducibility of the capsaicininduced dermal blood flow response as assessed by laser Doppler perfusion imaging. Br J Clin Pharmacol. 2007;64(5):580-90.

6. Vermeersch S, Benschop RJ, Van Hecken A, Monteith D, Wroblewski VJ, Grayzel D, et al. Translational pharmacodynamics of calcitonin gene-related peptide monoclonal antibody LY2951742 in a capsaicin-induced dermal blood flow model. J Pharmacol Exp Ther. 2015;354(3):350-7.

7. United States Adopted Name: erenumab; USAN File Number: (BC-146). Available from https://searchusan.ama-assn.org/ finder $/$ usan $/$ search $/ * /$ relevant $/ 1$; facet $=\% 20$ AND $\% 20$ Sponsor $\%$ 3A $\% 22$ Amgen $\% 20 I n c . \% 22$. [Website].

8. Shi L, Lehto S, Zhu DX, Sun H, Zhang J, Smith BP, et al. Pharmacological characterization of AMG 334, a potent and selective human monoclonal antibody against the calcitonin generelated peptide (GGRP) receptor. J Pharmacol Exp Ther. 2015;356:223-31.

9. de Hoon JN, Van Hecken A, Yan L, Smith B, Chen J, Bautista E, et al. Single-dose and multiple dose, phase 1, randomized, doubleblind, placebo-controlled studies of AMG 334 in healthy subjects and migraine patients. Headache. 2015;55(S3):Abstract PS35.

10. Levy G. Pharmacologic target-mediated drug disposition. Clin Pharmacol Ther. 1994;56(3):248-52.

11. Wang W, Wang EQ, Balthasar JP. Monoclonal antibody pharmacokinetics and pharmacodynamics. Clin Pharmacol Ther. 2008;84(5):548-58.

12. Ascending multiple-doses of AMG 334 in healthy subjects and in migraine patients (GlinicalTrials.gov Identifier: NCT01723514). Available at: https://clinicaltrials.gov/ct2/show/study/ NCT01723514?term=AMG+334\&rank=5. [Website].

13. Ascending single doses of AMG 334 in healthy subjects and migraine patients (ClinicalTrials.gov Identifier: NCT01723514). Available at: https://clinicaltrials.gov/ct2/show/NCT01688739? term $=\mathrm{AMG}+334 \&$ rank=2. [Website].

14. Gibiansky L, Gibiansky E, Kakkar T, Ma P. Approximations of the target-mediated drug disposition model and identifiability of model parameters. J Pharmacokinet Pharmacodyn. 2008;35(5):573-91.

15. Mager DE, Jusko WJ. General pharmacokinetic model for drugs exhibiting target-mediated drug disposition. J Pharmacokinet Pharmacodyn. 2001;28(6):507-32.

16. Ma P. Theoretical considerations of target-mediated drug disposition models: simplifications and approximations. Pharm Res. 2012;29(3):866-82.

17. Lobo ED, Hansen RJ, Balthasar JP. Antibody pharmacokinetics and pharmacodynamics. J Pharm Sci. 2004;93(11):2645-68.

18. Dirks NL, Meibohm B. Population pharmacokinetics of therapeutic monoclonal antibodies. Clin Pharmacokinet. 2010;49(10):633-59.

19. Sinclair SR, Kane SA, Van der Schueren BJ, Xiao A, Willson KJ, Boyle J, et al. Inhibition of capsaicin-induced increase in dermal 
blood flow by the oral CGRP receptor antagonist, telcagepant (MK-0974). Br J Clin Pharmacol. 2010;69(1):15-22.

20. Li CG, Vermeersch S, Denney WS, Kennedy WP, Palcza J, Gipson A, et al. Characterizing the PK/PD relationship for inhibition of capsaicin-induced dermal vasodilatation by MK-3207, an oral calcitonin gene related peptide receptor antagonist. Br J Clin Pharmacol. 2015;79(5):831-7.

21. Van der Schueren BJ, Rogiers A, Vanmolkot FH, Van Hecken A, Depre M, Kane SA, et al. Calcitonin gene-related peptide8-37 antagonizes capsaicin-induced vasodilation in the skin: evaluation of a human in vivo pharmacodynamic model. J Pharmacol Exp Ther. 2008;325(1):248-55.

22. Baker B, Smith J. A single dose, placebo-controlled randomized ascending dose study of ALD403, a humanized anti-calcitonin gene-related peptide monoclonal antibody administered IV or $\mathrm{SC}$ - pharmacokinetic and pharmacodynamics results. Cephalalgia. 2015;35(6S) Abstract PO082
23. Sun H, Dodick DW, Silberstein S, Goadsby PJ, Reuter U, Ashina M, et al. Safety and efficacy of AMG 334 for prevention of episodic migraine: a randomised, double-blind, placebo-controlled, phase 2 trial. Lancet Neurol. 2016;15(4):382-90.

24. Buntinx L, Vermeersch S, de Hoon J. Development of antimigraine therapeutics using the capsaicin-induced dermal blood flow model. Br J Clin Pharmacol. 2015;80(5):992-1000.

25. Tabrizi M, Bornstein GG, Suria H. Biodistribution mechanisms of therapeutic monoclonal antibodies in health and disease. AAPS J. 2010;12(1):33-43.

26. Hostetler ED, Joshi AD, Sanabria-Bohorquez S, Fan H, Zeng Z, Purcell M, et al. In vivo quantification of calcitonin gene-related peptide receptor occupancy by telcagepant in rhesus monkey and human brain using the positron emission tomography tracer [11C]MK-4232. J Pharmacol Exp Ther. 2013;347(2):478-86. 